\title{
Eugene C. Lin, Edward J. Escott, Kavita D. Garg, Andrew G. Bleicher, David Alexander: Practical differential diagnosis for CT and MRI
}

\author{
Thieme, Stuttgart, 2008. ISBN: 978-1-58890-655-7
}

\author{
Maurizio Sacco • Luigi Mansi
}

Published online: 11 July 2009

(C) Springer-Verlag 2009

The increased availability of CT scans and magnetic resonance imaging to an ever higher number of radiologists within the last decade has determined a growing need to resolve daily diagnostic and technology-related problems.

In fact, the presence in the same department of both procedures creates the need to have a clear evidence of relative advantages and disadvantages to support the choice of the most cost-effective technique in each pathological condition and/or in the single patient to be evaluated.

Consequently, we have witnessed an exponential growth in the number of publications and complex specialty texts dedicated to diagnostic imaging, sometimes with a redundant effect creating confusion more than useful information.

In this sense we strongly appreciated the editorial idea of this publication. The authors have succeeded in creating a dynamic and easily readable text so much so that we believe its ideal place is beside the radiologist's workstation of image analysis. In fact, the outline of this volume is pragmatic and simple for which it is directed specially to the radiologist who still has a vast preparation in image interpretation.

The format does not try to furnish findings and data of every pathological state nor does it attempt to provide notions of aetiopathology; rather it directly aims at the

M. Sacco $(\bowtie) \cdot$ L. Mansi

Naples, Italy

e-mail: ATAUALPAMASA@libero.it

L. Mansi

e-mail: luigi.mansi@unina2.it selection and analysis of radiologic signs and of the particular radiologic expression of the disease. It means that this book can be helpful in a fast and accurate decision on the procedure to be chosen to answer to the most frequent pathological questions. Conversely, it cannot be used as a unique reference for experts who wants to find information on the whole field of interest concerning clinical applications of CT and MRI.

The structure of the text is primarily organised in sections relating to the most important fields where a differential diagnosis can be achieved with both procedures, i.e. brain, head, neck, lung, abdomen, pelvis, musculoskeletal system and spinal cord. The presentation by the author's of a list of similar diagnostic image findings as well as a description of characteristics that aid in distinguishing different pathological states that share similar findings is interesting, also from the didactic point of view. It creates clear evidence of the prevalence of one approach in respect to the other, simultaneously giving evidence that the technique with minor capabilities for that specific purpose can however be used to give effective information in the absence of the first choice methodology.

In conclusion, we suggest this book as a useful publication for the library of all diagnostic imaging departments. The major interest is for expert radiologists involved in decisions concerning the construction of the most effective diagnostic tree for the single patient and/or for the most productive schedule in diagnostic imaging departments. The book is also interesting for residents and young radiologists to grow in their confidence to reliably make technological and methodological choices. 